\title{
Fourier inversion formulas in option pricing and insurance
}

\author{
Daniel Dufresne ${ }^{1}$, Jose Garrido ${ }^{2}$ and Manuel Morales ${ }^{3}$ \\ ${ }^{1}$ Centre for Actuarial Studies, \\ University of Melbourne, Australia \\ ${ }^{2}$ Department of Mathematics and Statistics, \\ Concordia University, Montreal, Canada \\ ${ }^{3}$ Department of Mathematics and Statistics, \\ Université de Montréal, Canada
}

December 21, 2005

\begin{abstract}
Several authors have used Fourier inversion to compute option prices. In insurance, the expected value of $\max (S-K, 0)$ also arises in excess-of-loss or stop-loss insurance, and similar techniques may be used. Lewis (2001) used Parseval's theorem to find formulas for option prices in terms of the characteristic function of the log-price. This paper aims at taking the same idea further: (1) formulas requiring weaker assumptions; (2) relationship with classical inversion theorems; (3) formulas for payoffs which occur in insurance.
\end{abstract}

\section{Introduction}

Lewis (2001) gives formulas which price options without having first to find the distribution of the underlying, by applying Parseval's theorem. All that is needed is the characteristic function (= Fourier transform) of the logarithm of underlying and the Fourier transform of the payoff function. Fourier methods are applied to option pricing in several other papers, for instance Bakshi \& 
Madan (2000), Carr \& Madan (1999), Heston (1993), Lee (2004), Raible (2000).

In insurance, the payoff

$$
(S-K)_{+}=\max (S-K, 0)
$$

also occurs in excess-of-loss or stop-loss contracts, so Parseval's theorem might also be used to find the pure premiums. This paper explores the computation of both option prices and insurance premiums via Parseval's theorem in a unified setting.

The mathematical problem is the same in insurance as in option pricing, that is, the computation of $\mathbb{E} g(S)$ for some function $g(\cdot)$. The difference is that in many cases option pricing models focus on the logarithm of $S$ (the "log-price"), while insurance applications are usually phrased in terms of the distribution of $S$ itself. For instance, the Black-Scholes formula for a call option is the expectation of the payoff $(S-K)_{+}$, where $\log S$ has a normal distribution; more recent models also specify the distribution of the log-price, rather than $S$ itself. The consequence is that the Fourier transform which is likely to be known is that of $X=\log S$. This explains the particular form of the formulas in Lewis (2001).

By contrast, in insurance applications $S$ is often (though not always) a distribution for which the Fourier transform $\mathbb{E} \exp (i u S)$ is known. This is why we will identity two different classes of inversion formulas: (1) those where the Fourier transform of $\log (S)$ is known (and thus appears in the inversion formula), and (2) those where the Fourier transform of $\log (S)$ appears. The first kind of inversion formula will be referred to as "Mellin-type", since it is the Mellin transform $\mathbb{E} \exp (i u \log (S))=\mathbb{E} S^{i u}$ which is used, and the other kind will be called "Fourier-type". The formulas in Lewis (2001) are thus all of Mellin type, while the insurance examples of Section 4 are all of Fourier type. We do not suggest that this classification is essential, or that it neatly differentiates option pricing from insurance (it does not), but we found it useful in presenting a unified view of the applications of Parseval's theorem to option pricing and insurance.

Section 2 states the particular form of the Parseval theorem we will use, and recalls two standard theorems of probability theory which are directly related to the pricing formulas which follow. Section 3 gives the main results of the paper. Lewis (2001) gives formulas which require the finiteness of $\mathbb{E} S^{\alpha}$ for some $\alpha$ different from 0 ; this is good enough in many cases, but not 
always feasible. We give general formulas which do not require this type of assumptions (this is where our formulas are reminiscent of the classical inversion formulas for distribution functions). Section 4 gives some applications. (N.B. This working paper does not contain all the numerical computations.) The appendices contain some background on Fourier transforms and also the longer proofs.

Notation. We denote $F_{X}(x)=\mathbb{P}\{X \leq x\}$ the distribution function of $X$. $\mu_{X}$ is the measure on $\mathbb{R}$ induced by a random variable $X$, that is, $\mu_{X}(B)=$ $\mathbb{P}\{X \in B\}, B$ a Borel subset of $\mathbb{R}$. The Fourier transform of a function $f: \mathbb{R} \rightarrow \mathbb{C}$ (when it exists) is denoted

$$
\hat{f}(u)=\int_{\mathbb{R}} e^{i u x} f(x) d x .
$$

The Fourier transform (often called the characteristic function) of the distribution of $X$ is denoted

$$
\hat{\mu}_{X}(u)=\int_{\mathbb{R}} e^{i u x} \mu_{X}(d x)=\mathbb{E} e^{i u X} .
$$

\section{Preliminaries}

\subsection{Parseval's theorem}

Theorem A.6 (Parseval's theorem, see Appendix A) says that

$$
\mathbb{E} g(X)=\frac{1}{2 \pi} P V \int_{-\infty}^{\infty} \hat{g}(-u) \hat{\mu}_{X}(u) d u
$$

if, among other things, the function $g$ is integrable. This is not the case for the functions

$$
g_{1}(x)=\left(e^{x}-K\right)_{+} \quad \text { or } \quad g_{2}(x)=\left(K-e^{x}\right)_{+},
$$

and Theorem A.6 cannot be applied directly. Lewis (2001) proposed to work around this problem by using an exponential damping factor. For any function $\varphi$, let

$$
\varphi^{\alpha}(x)=e^{\alpha x} \varphi(x), \quad x \in \mathbb{R} .
$$

The Fourier transform of $\varphi^{\alpha}$ will be denoted $\widehat{\varphi^{\alpha}}$. 
If $X$ has a probability density function $f_{X}$, and if it happens that both $g^{-\alpha}$ and $f_{X}^{\alpha}$ are in $L^{1}$, then Theorem A.6 implies

$$
\mathbb{E} g(X)=\int_{-\infty}^{\infty} g^{-\alpha}(x) f_{X}^{\alpha}(x) d x=\frac{1}{2 \pi} P V \int_{-\infty}^{\infty} \widehat{g^{-\alpha}}(-u) \widehat{f_{X}^{\alpha}}(u) d u .
$$

It turns out that the payoff functions $g_{1}$ and $g_{2}$ above are integrable when multiplied by suitable exponential functions, and thus Parseval's identity can be used to compute option prices, provided that $f_{X}^{\alpha}$ is integrable.

Lewis (2001) assumed that $X$ has a probability density function; we reformulate his idea by removing this asssumption, as it does not hold in all applications. For a signed measure $\mu$ on $\mathbb{R}$ and $\alpha \in \mathbb{R}$, define a new signed measure $\mu^{\alpha}$ by

$$
\mu^{\alpha}(d x)=e^{\alpha x} \mu(d x) .
$$

Then the Fourier transform of $\mu_{X}^{\alpha}$ is

$$
\widehat{\mu_{X}^{\alpha}}(u)=\mathbb{E} e^{(i u+\alpha) X}=\hat{\mu}_{X}(u-i \alpha) .
$$

Theorem A.6 immediately yields the following result, which will be used in the rest of this paper.

Theorem 2.1 Let $X$ be a random variable, and suppose that for a particular $\alpha \in \mathbb{R}$,

(a) $\mathbb{E}\left(e^{\alpha X}\right)<\infty$,

(b) $g^{-\alpha} \in L^{1}$,

(c) the function $y \mapsto \mathbb{E} g(y+X)$ is continuous at the origin and satisfies condition (b) of Theorem A.5.

Then

$\mathbb{E} g(X)$

$=\frac{1}{2 \pi} P V \int_{-\infty}^{\infty} \widehat{g^{-\alpha}}(-u) \widehat{\mu_{X}^{\alpha}}(u) d u=\frac{1}{2 \pi} P V \int_{-\infty}^{\infty} \hat{g}(-u+i \alpha) \hat{\mu}_{X}(u-i \alpha) d u$.

(N.B. The function $y \mapsto \mathbb{E} g(y+X)$ is not always continuous, for instance, consider $X \equiv 1$ and $g(x)=I_{\{x>1\}}$.)

The following lemmas give sufficient conditions for condition (b) of Theorem A.5 to hold. 
Lemma 2.1 Condition (b2) of Theorem A.6 is satisfied if $g$ has bounded variation.

Proof. Define $G(y)=\mathbb{E} g(y+X)$ and let $V^{g}$ be the variation of $g(\cdot)$ over $\mathbb{R}$. If $\left\{y_{j}\right\}$ is an increasing sequence. Then

$$
\begin{aligned}
\sum_{j}\left|\Delta G\left(y_{j}\right)\right| & \leq \int \sum_{j}\left|g\left(y_{j}+x\right)-g\left(y_{j-1}+x\right)\right| d \mu_{X}(x) \\
& \leq \int V^{g} d \mu_{X}(x)=V^{g}<\infty .
\end{aligned}
$$

Lemma 2.2 (a) Suppose that there are $a_{1}<a_{2}<\cdots<a_{n}$ such that

(i) $\mathbb{P}\left\{X=a_{j}\right\}=0$ for $j=1, \ldots, n$,

(ii) $g(\cdot)$ is uniformly bounded and piecewise continuous over $\left(-\infty, a_{1}\right),\left(a_{1}, a_{2}\right)$, $\cdots,\left(a_{n-1}, a_{n}\right),\left(a_{n}, \infty\right)$, and has finite limits $g\left(a_{j}-\right), g\left(a_{j}+\right)$.

Then $G(y)=\mathbb{E} g(y+X)$ is continuous at $y=0$.

(b) If $\mathbb{E} X_{+}<\infty$, then $G(y)=\mathbb{E}(y+X-K)_{+}$is continuous at $y=0$.

Proof. (a) Write

$$
\mathbb{E} g(y+X)=\sum_{k=1}^{n+1} \mathbb{E}\left[g(y+X) I_{k}(X)\right],
$$

where $\left\{I_{k}(\cdot)\right\}$ are the indicator functions of the intervals $\left(-\infty, a_{1}\right),\left(a_{1}, a_{2}\right)$, $\ldots,\left(a_{n-1}, a_{n}\right),\left(a_{n}, \infty\right)$. Then, as $y \rightarrow 0$,

$$
g(y+X(\omega)) I_{k}(X(\omega)) \rightarrow g(X(\omega)) I_{k}(X(\omega)),
$$

for all $\omega$. Since $g$ is uniformly bounded,

$$
\mathbb{E}\left[g(y+X) I_{k}(X)\right] \rightarrow \mathbb{E}\left[g(X(\omega)) I_{k}(X)\right],
$$

by dominated convergence, which yields the result.

To prove part (b), observe that $(y+S-K)_{+} \leq S_{+}+(y-K)_{+}$and the result follows by dominated convergence. 


\subsection{Two classical theorems}

We present two standard theorems which are intimately related to the option or stop-loss formulas which follow. Each expresses the distribution function of a random variable as a Fourier inversion integral. The best known proofs of these results (see Lucaks, 1970, p.31, and Kendall \& Stuart, 1977, p.97) rely on Dirichlet integrals, but Appendix B gives proofs based on Parseval's theorem.

Theorem 2.2 Ig $a$ and $a+h$ are continuity points of $F_{X}(\cdot)$, then

$$
F_{X}(a+h)-F_{X}(a)=\frac{1}{2 \pi} P V \int_{-\infty}^{\infty} \frac{1-e^{-i u h}}{i u} e^{-i u a} \hat{\nu}_{X}(u) d u
$$

Theorem 2.3 If $F_{X}(\cdot)$ is continuous at $x=b$, then

$$
F_{X}(b)=\frac{1}{2}+\frac{1}{2 \pi} P V \int_{0}^{\infty} \frac{1}{i u}\left[e^{i u b} \hat{\nu}_{X}(-u)-e^{-i u b} \hat{\nu}_{X}(u)\right] d u .
$$

In option pricing, Theorem 2.3 leads to the well-known formula

$$
\mathbb{E}\left(e^{X}-K\right)_{+}=\mathbb{E}\left(e^{X}\right) \Pi_{1}-K \Pi_{2},
$$

where

$$
\begin{aligned}
& \Pi_{1}=\mathbb{E}\left[e^{X} \mathbf{1}_{\left\{e^{X}>K\right\}}\right] / \mathbb{E}\left(e^{X}\right)=\frac{1}{2}+\frac{1}{\pi} \int_{0}^{\infty} \operatorname{Re}\left[\frac{K^{-i u} \hat{\nu}_{X}(u-i)}{i u \hat{\nu}_{X}(-i)}\right] d u \\
& \Pi_{2}=\mathbb{P}\left\{e^{X}>K\right\} .
\end{aligned}
$$

\subsection{Mellin-type and Fourier-type formulas}

Lewis (2001) considers payoffs which are explicit functions of $e^{X}$, such as the usual call and put payoffs $g_{1}$ and $g_{2}$ in (2.1). This is because most financial models are expressed in terms of the log-price. For instance, a formula for $\mathbb{E}(S-K)_{+}$is obtained in terms of

$$
\mathbb{E} e^{i u \log S}=\mathbb{E} S^{i u} .
$$


The insurance applications considered in Section 4, however, lead to expressions of the type $\mathbb{E}(S-K)_{+}$, but the inversion formulas are in terms of the Fourier transform $\mathbb{E}\left(e^{i u S}\right)$.

The expression in Eq.(2.2) is known as the Mellin transform of the distribution of $S$. In order to distinguish these two situations, we will call "Mellin-type" the formulas where $\mathbb{E} S^{i u}$ appears, and "Fourier-type" those where $\mathbb{E}\left(e^{i u S}\right)$ appears.

\section{Inversion formulas}

In this section, formulas are derived for the expectations of the payoffs $g_{1}$ and $g_{2}$ in (2.1). In each case, Parseval's theorem yields an inversion integral along the line $u-i \alpha$ in the complex plane, if $\alpha$ can be found such that (i) $g^{-\alpha}$ is in $L^{1}$ and (ii) $\mathbb{E} \exp (\alpha X)$ is finite. It is not always possible to find such $\alpha$, depending on the function $g$ considered and also the distribution of $X$. For this reason, we derive general formulas which do not assume that such $\alpha \neq 0$ exists. In each case the approach is the same: truncate the distribution of $X$ in such a way that Parseval's theorem applies for some $\alpha \neq 0$; next, let $\alpha$ tend to 0 , and, finally, remove the truncation of the distribution of $X$. The similarity of these general "no- $\alpha$ " formulas with the classical theorems of Section 2 will be noted.

An important point to keep in mind in what follows is that if there is $\alpha>0$ such that $\mathbb{E} \exp (\alpha X)<\infty$, then necessarily $\mathbb{E} \exp \left(\alpha^{\prime} X\right)<\infty$ for $0<\alpha^{\prime}<\alpha$ (the same applies for $\alpha<0$ ). The set of $\alpha$ such that $g^{-\alpha} \in L^{1}$, when it exists, is either an interval or a single point. Hence, the set of $\alpha$ such that both $\mathbb{E} \exp (\alpha X)<\infty$ and $g^{-\alpha} \in L^{1}$ is either empty or an interval (possibly reduced to a single point). This has numerical implications, since the observed accuracy of the integral formula often varies with $\alpha$ within the allowed interval.

\subsection{Mellin-type formulas}

The proof of the next theorem can be found in Appendix B.

Theorem 3.1 Let $S \geq 0, K>0$ and

$$
h(u)=\frac{K^{-i u+1}}{i u(i u-1)} \mathbb{E}\left(S^{i u}\right) .
$$


(a) If there exists $\alpha<0$ such that $\mathbb{E}\left(S^{\alpha}\right)<\infty$, then

$$
\mathbb{E}(K-S)_{+}=K \mathbb{P}\{S=0\}+\frac{1}{2 \pi} P V \int_{-\infty}^{\infty} h(u-i \alpha) d u
$$

If, moreover, $\mathbb{E}(S)<\infty$, then

$$
\mathbb{E}(S-K)_{+}=\mathbb{E} S-K[1-\mathbb{P}\{S=0\}]+\frac{1}{2 \pi} P V \int_{-\infty}^{\infty} h(u-i \alpha) d u .
$$

(b) In all cases,

$$
\mathbb{E}(K-S)_{+}=\frac{K}{2}[1+\mathbb{P}\{S=0\}]+\frac{1}{\pi} P V \int_{0}^{\infty} \operatorname{Re}[h(u)] d u .
$$

If $\mathbb{E}(S)<\infty$,

$$
\mathbb{E}(S-K)_{+}=\mathbb{E} S-\frac{K}{2}[1-\mathbb{P}\{S=0\}]+\frac{1}{\pi} P V \int_{0}^{\infty} \operatorname{Re}[h(u)] d u .
$$

\subsubsection{Confirmation in the case where $\mathrm{S}$ has a discrete distribution}

Suppose that $X \equiv x_{0}$. This means that $\hat{\mu}_{X}(u)=e^{i u x_{0}}$, and

$$
h(u)=-\frac{e^{c} e^{i u\left(x_{0}-c\right)}}{i u(1-i u)} .
$$

Then

$$
\operatorname{Re}[h(u)]=-\frac{e^{c} \cos (u \beta)}{1+u^{2}}-\frac{e^{c} \sin (u \beta)}{u\left(1+u^{2}\right)}, \quad \beta=x_{0}-c .
$$

Now

$$
\frac{1}{\pi} \int_{0}^{\infty} \frac{\cos (u \beta)}{1+u^{2}} d u=\frac{1}{2} \int_{-\infty}^{\infty} \frac{e^{i u \beta}}{\pi\left(1+u^{2}\right)} d u=\frac{1}{2} e^{-|\beta|}
$$

(this is $\frac{1}{2}$ times the characteristic function of the Cauchy distribution). Also, letting $\operatorname{sign} \beta=I_{\{x>0\}}-I_{\{x<0\}}$,

$$
\begin{aligned}
\int_{0}^{\infty} \frac{\sin (u \beta)}{u\left(1+u^{2}\right)} d u & =\int_{0}^{\infty} \int_{0}^{\beta} \frac{\cos (u y)}{1+u^{2}} d y d u \\
& =\int_{0}^{\beta} \frac{1}{2} e^{-|y|} d y=(\operatorname{sign} \beta) \frac{1}{2}\left(1-e^{-|\beta|}\right) .
\end{aligned}
$$


Hence,

$$
\begin{aligned}
\frac{e^{c}}{2}+\frac{1}{\pi} \int_{0}^{\infty} \operatorname{Re}[h(u)] d u & =\frac{e^{c}}{2}-\frac{e^{c}}{2}\left[e^{-|\beta|}+(\operatorname{sign} \beta)\left(1-e^{-|\beta|}\right)\right] \\
& =\frac{e^{c}}{2}[1-(\operatorname{sign} \beta)]\left(1-e^{-|\beta|}\right)=\left(e^{c}-e^{x_{0}}\right)_{+} .
\end{aligned}
$$

Since a discrete distribution is a combination of degenerate distributions, this shows that the formula is correct for discrete random variables $S>0$. It is true for any $S \geq 0$, because if $\mathbb{P}\{S=0\}=1$, then

$$
\mathbb{E}(K-S)_{+}=K
$$

while

$$
K+\frac{1}{2 \pi} \int_{0}^{\infty} \frac{e^{c}}{i u}\left[e^{i u c} \frac{\mathbb{E}\left(0^{-i u}\right)}{1+i u}-e^{-i u c} \frac{\mathbb{E}\left(0^{i u}\right)}{1-i u}\right] d u=K
$$

\subsection{Fourier-type formulas}

We now look at formulas for the payoffs

$$
g_{3}(x)=(x-K)_{+}, \quad g_{4}(x)=(K-x)_{+} I_{\{0 \leq x \leq K\}}
$$

in terms of $\hat{\mu}_{X}(u)=\mathbb{E}\left(e^{i u X}\right)$. Exponential damping factors $e^{\alpha x}$ may be used just as in the previous section, but we show that one may also use polynomial damping factors.

\subsubsection{Exponential damping factors}

Theorem 3.2 Let $X \geq 0$.

(a) If there exists $\alpha>0$ such that $\mathbb{E}\left(e^{\alpha X}\right)<\infty$, then $\mathbb{E}(X-K)_{+}<\infty$ for $K \in \mathbb{R}$ and

$$
\mathbb{E}(X-K)_{+}=\frac{1}{2 \pi} P V \int_{-\infty}^{\infty} \hat{g}_{3}(-u+i \alpha) \hat{\mu}_{X}(u-i \alpha) d u
$$

where

$$
\hat{g}_{3}(z)=-\frac{e^{i z K}}{z^{2}}
$$


(b) For any $\alpha \in \mathbb{R}$ such that $\mathbb{E}\left(e^{\alpha X}\right)<\infty$ (including $\alpha=0$ ) and $K \geq 0$,

$$
\mathbb{E}(K-X)_{+}=\frac{1}{2 \pi} P V \int_{-\infty}^{\infty} \hat{g}_{4}(-u+i \alpha) \hat{\mu}_{X}(u-i \alpha) d u,
$$

where

$$
\hat{g}_{4}(z)=\frac{1}{z^{2}}\left(1+i z K-e^{i z K}\right) .
$$

Proof. Part (a) is a direct application of Parseval's theorem and Lemma 2.2 , given that if $\operatorname{Im}(z)>0$, then, for any $K$,

$$
\hat{g}_{3}(z)=\int_{K}^{\infty}(x-K) e^{i z x} d x=e^{i z K} \int_{0}^{\infty} y e^{i z y} d y=-\frac{e^{i z K}}{z^{2}} .
$$

For part (b), it is clear that $g_{4}^{-\alpha} I_{[0, K]} \in L^{1}$ for any $\alpha \in \mathbb{R}$; also, $g_{4}$ is continuous everywhere except at $x=0$. Provided $\hat{\mu}_{X}(-i \alpha)<\infty$, we can thus apply Parseval's theorem and Lemma 2.2, with

$$
\hat{g}_{4}(z)=\int_{0}^{\infty}\left(g_{3}(x)+K-x\right) e^{i z x} d x=\frac{1}{z^{2}}\left(1+i z K-e^{i z K}\right) .
$$

In Section 3.2.3, a variation on part (a) of this theorem is given which does not require that $\mathbb{E}\left(e^{\alpha X}\right)<\infty$ for any $\alpha>0$.

\subsubsection{Polynomial damping factors}

An alternative to the formulas in Theorem 3.2 is to use a polynomial damping factor. For $\beta \in\{1,2, \ldots\}$ and $c>0$, let

$$
g^{[-\beta]}(x)=(1+c x)^{-\beta} g(x), \quad d \mu_{X}^{[\beta]}(x)=(1+c x)^{\beta} d \mu_{X}(x) .
$$

For the payoff $g_{3}(x)=(x-K)_{+}$, given $\beta \geq 2$,

$\widehat{g_{3}^{[-\beta]}}(u)=\int_{\mathbb{R}} \frac{e^{i u x}(x-K)_{+}}{(1+c x)^{\beta}} d x=\frac{e^{i u K}}{c^{2}(1+c K)^{\beta-2}} \Psi(2,3-\beta,-i u(1+c K) / c)$,

where

$$
\Psi(\alpha, \gamma, z)=\frac{1}{\Gamma(\alpha)} \int_{0}^{\infty} \frac{e^{-z t} t^{\alpha-1}}{(1+t)^{\alpha-\gamma+1}} d t, \quad \alpha>0
$$


is the confluent hypergeometric function of the second kind. (The integral formula above holds (i) for $\operatorname{Re}(z)>0$ and also (ii) for $\operatorname{Re}(z)=0, \operatorname{Im}(z) \neq 0$ if $\gamma \leq 1$; for more details, see Lebedev, 1972, Chapter 9).

The function $\Psi$ above may be expressed in terms of the incomplete gamma function; since

$$
\Psi(2,3-\beta, z)=\Psi(1,3-\beta, z)-\Psi(1,2-\beta, z),
$$

the formula in (3.1) may be written in terms of

$$
\Psi(1, \gamma, z)=z^{1-\gamma} e^{z} \Gamma(\gamma-1, z)
$$

where

$$
\Gamma\left(a, z_{0}\right)=\int_{z_{0}}^{\infty} x^{a-1} e^{-x} d x, \quad\left|\arg \left(z_{0}\right)\right|<\pi .
$$

Because $\beta$ is a positive integer, an alternative is to use integration by parts to show that, if $n=0,1,2,3, \ldots$,

$$
\Psi(1,-n, z)=\sum_{j=0}^{n} \frac{(-z)^{j}}{(n+1-j)_{j+1}}+\frac{(-z)^{n+1}}{(n+1) !} \Psi(1,1, z), \quad \operatorname{Re}(z) \geq 0 .
$$

The remaining hypergeometric function $\Psi(1,1, z)$ may in turn be written as an incomplete gamma function (see (3.2)), or else as

$$
\Psi(1,1, z)=-e^{z} E_{1}(-z), \quad E_{1}(z)=-\int_{-z}^{\infty} \frac{e^{-t}}{t} d t, \quad|\arg (-z)|<\pi .
$$

Here $E_{1}(\cdot)$ is the exponential integral function. For more details on the special functions above, see Abramowitz and Stegun (1970) or Lebedev (1972).

For $\beta \geq 2$, the Fourier transform of

$$
g_{4}^{[-\beta]}(x)=(1+c x)^{-\beta}(K-x)_{+}
$$

may be found in the obvious way: since

$$
g_{4}^{[-\beta]}(x)=g_{3}^{[-\beta]}(x)+(1+c x)^{-\beta}(K-x),
$$

we get

$$
\begin{aligned}
\widehat{g_{4}^{[-\beta]}}(u) & =\widehat{g_{3}^{[-\beta]}}(u)+\int_{0}^{\infty} \frac{e^{i u x}(K-x)}{(1+c x)^{\beta}} d x \\
& =\widehat{g_{3}^{[-\beta]}}(u)+\left(\frac{K}{c}+\frac{1}{c^{2}}\right) \Psi(1,2-\beta,-i u / c)-\frac{1}{c^{2}} \Psi(1,3-\beta,-i u / c) .
\end{aligned}
$$


The case $\beta=1$ is different:

$\widehat{g_{4}^{[-1]}}(u)=\frac{c K+1}{c^{2}}\left[\Psi(1,1,-i u / c)-e^{i u K} \Psi(1,1,-i u(1+c K) / c)\right]-\frac{1}{i u c}\left(e^{i u K}-1\right)$.

As to the Fourier transform of $\mu_{X}^{[\beta]}(x)$,

$$
\widehat{\mu_{X}^{[\beta]}}(u)=\sum_{k=0}^{\beta}\left(\begin{array}{l}
\beta \\
k
\end{array}\right) c^{k} \int_{\mathbb{R}} x^{k} e^{i u x} d \mu_{X}(x)=\sum_{k=0}^{\beta}\left(\begin{array}{l}
\beta \\
k
\end{array}\right)(-c i)^{k} \frac{\partial^{k}}{\partial u^{k}} \hat{\mu}_{X}(u) .
$$

Recall that if $\mathbb{E} X^{k}<\infty$, then $\frac{\partial^{k}}{\partial u^{k}} \hat{\mu}_{X}(u)$ exists for all $u \in \mathbb{R}$.

Theorem 3.3 Let $X \geq 0, c>0$.

(a) If $\mathbb{E} X^{\beta}<\infty$ and $\beta \in\{2,3, \ldots\}$,

$$
\mathbb{E}(X-K)_{+}=\frac{1}{2 \pi} P V \int_{-\infty}^{\infty} \widehat{g_{3}^{[-\beta]}}(u)\left[\sum_{k=0}^{\beta}\left(\begin{array}{l}
\beta \\
k
\end{array}\right) \beta^{k}(-c i)^{k} \frac{\partial^{k}}{\partial u^{k}} \hat{\mu}_{X}(u)\right] d u .
$$

(b) If $\mathbb{P}\{X=0\}=0, \mathbb{E} X^{\beta}<\infty, c>0$ and $\beta \in\{0,1,2,3, \ldots\}$,

$$
\mathbb{E}(K-X)_{+}=\frac{1}{2 \pi} P V \int_{-\infty}^{\infty} \widehat{g_{4}^{[-\beta]}}(u)\left[\sum_{k=0}^{\beta}\left(\begin{array}{l}
\beta \\
k
\end{array}\right) \beta^{k}(-c i)^{k} \frac{\partial^{k}}{\partial u^{k}} \hat{\mu}_{X}(u)\right] d u .
$$

\subsubsection{Ladder height distributions}

In this section, we show how an inversion formula can be found for $\mathbb{E}(X-K)_{+}$ as a direct application of Theorem 2.3. We know that, for any $X \geq 0$,

$$
\mathbb{E}(X-K)_{+}=\int_{K}^{\infty} \mathbb{P}(X>y) d y .
$$

Define a new distribution (sometimes called the "ladder height" distribution associated with $X$ ) with density

$$
f_{\bar{X}}(x)=\frac{\mathbb{P}(X>x)}{\mathbb{E} X} \mathbf{1}_{\{x>0\}}
$$

Then

$$
\mathbb{E}(X-K)_{+}=(\mathbb{E} X) \mathbb{P}(\bar{X}>K) .
$$


Theorem 3.4 If $X \geq 0, \mathbb{E}(X)<\infty$ and $K \geq 0$,

$$
\begin{aligned}
\mathbb{E}(X-K)_{+} & =\frac{\mathbb{E} X}{2}+\frac{1}{\pi} P V \int_{0}^{\infty} \operatorname{Re}\left[\frac{e^{-i u K}\left(\hat{\mu}_{X}(u)-1\right)}{(i u)^{2}}\right] d u \\
& =\frac{1}{2 \pi} P V \int_{-\infty}^{\infty} \frac{e^{-i u K}\left[\hat{\mu}_{X}(u)-1-i u \mathbb{E}(X)\right]}{(i u)^{2}} d u .
\end{aligned}
$$

Proof. An easy calculation yields

$$
\hat{\mu}_{\bar{X}}(u)=\frac{\hat{\mu}_{X}(u)-1}{i u \mathbb{E} X} .
$$

Theorem 2.3 says that

$$
\mathbb{P}\{\bar{X}>K\}=\frac{1}{2}+\frac{1}{\pi} \int_{0}^{\infty} \operatorname{Re}\left[\frac{e^{-i u K} \hat{\mu}_{\bar{X}}(u)}{i u}\right] d u,
$$

which implies (3.3).

Next, apply the same idea to $\bar{X}$, to get

$$
\begin{aligned}
f \overline{\overline{\mathrm{X}}}(x) & =\frac{\mathbb{P}(\bar{X}>x)}{\mathbb{E} \bar{X}} \mathbf{1}_{\{x>0\}}=\frac{\mathbb{E}(X-x)_{+}}{\mathbb{E}\left(X^{2}\right) / 2} \\
\hat{\mu} \overline{\overline{\mathrm{X}}}(u) & =\frac{\hat{\mu}_{\bar{X}}(u)-1}{i u \mathbb{E} X}=\frac{\hat{\mu}_{\bar{X}}(u)-1-i u \mathbb{E} X}{(i u)^{2} \mathbb{E}\left(X^{2}\right) / 2}
\end{aligned}
$$

(this requires $\left.\mathbb{E} X^{2}<\infty\right)$. The function $f \overline{\overline{\mathrm{X}}}(\cdot)$ is integrable and differentiable; therefore, apply Theorem A.5 to get (3.4). To remove the assumption that $\mathbb{E}\left(X^{2}\right)<\infty$, truncate the distribution of $X$ by defining $X^{a}=X \wedge a$; formula (3.4) holds for $X^{a}$. Next, let $a$ tend to infinity. The integral is unchanged, because $\mathbb{E}\left(e^{i u X^{a}}\right)$ tends to $\mathbb{E}\left(e^{i u X}\right)$ uniformly in $u$.

The above formulas are similar to the one in part (a) of Theorem 3.2. The main difference is that Theorem 3.2(a) requires the additional assumption that $\mathbb{E}\left(e^{\alpha X}\right)<\infty$ for some $\alpha>0$. Note that even though all three formulas give the same result for $K \geq 0$, for $K<0$ they are different: Theorem 3.2(a) gives $\mathbb{E}(X)-K$, formula (3.3) yields $\mathbb{E}(X)$, and formula (3.4) yields 0 .

It is interesting to reconcile the preceding results with Theorem 3.2. To 
get (3.3), assume Theorem 3.2 holds, and write

$$
\begin{aligned}
\int_{-M}^{M} \hat{g}_{3}(-u+i \alpha) \hat{\mu}_{X}(u & -i \alpha) d u \\
& =-\int_{-M-i \alpha}^{M-i \alpha} \frac{e^{-i z K}}{z^{2}} \hat{\mu}_{X}(z) d z \\
& =-\int_{-M-i \alpha}^{M-i \alpha} \frac{e^{-i z K}}{z^{2}}\left(\hat{\mu}_{X}(z)-1\right) d z-\int_{-M-i \alpha}^{M-i \alpha} \frac{e^{-i z K}}{z^{2}} d z .
\end{aligned}
$$

The change of variable $z=M w$ shows that the last integral tends to 0 as $M \rightarrow \infty$. The path of integration in the remaining integral can be pushed up to the real axis, yielding (3.3) when $M$ tends to infinity (the pole at the origin leaves $\pi E(X))$. To get (3.4), proceed similarly:

$\frac{e^{-i z K}}{z^{2}} \hat{\mu}_{X}(z)=\frac{e^{-i z K}}{z^{2}}\left(\hat{\mu}_{X}(z)-1-i z \mathbb{E}(X)\right)+\frac{e^{-i z K}}{z^{2}}(1+i z \mathbb{E}(X))=(A)+(B)$.

The integral of $(A)$ tends to (3.4) as the path of integration is moved up to the real axis (with no residue), while the integral of $(B)$ over the segment $(-M-i \alpha, M-i \alpha)$ tends to 0 as $M$ tends to infinity.

It is possible to iterate the procedure which led to (3.4). Each iteration yields a new inversion formula of the form

$\mathbb{E}(X-K)_{+}$

$$
=\frac{1}{2 \pi} \int_{-\infty}^{\infty} \frac{e^{-i u K}\left[\hat{\mu}_{X}(u)-1-i u \mathbb{E}(X)-\cdots-(i u)^{n} \mathbb{E}\left(X^{n}\right) / n !\right]}{(i u)^{2}} d u
$$

for $K>0$ and $n=2,3, \ldots$, but it is unclear whether there is any benefit in using these formulas. (Observe that these are formulas for the higher order derivatives of the ladder height densities, and that they vanish for $K<0$. Numerical problems may be experienced for $K$ close to 0 , since for $n \geq 2$ the theoretical value of the inversion integral involves the Dirac function and its derivatives at $K=0$.)

\section{Examples}

In the first two examples (compound Poisson/exponential, generalized Pareto) there are closed form expressions for the expected payoffs as well as for Fourier 
and Mellin transforms. It is therefore possible to test the inversion formulas derived above against the exact expected payoffs. In the other examples (compound Poisson/Pareto, compound Poisson/Pareto plus $\alpha$-stable), there are no closed form expressions for the expected payoffs we consider, and simulation is used to assess the performance of the Fourier inversion formulas. (N.B. The numerical illustrations are incomplete.)

\subsection{Compound Poisson/Exponential distribution}

In this example, the explicit distribution is known, as well as both the Fourier and Mellin transforms. We will show that this distribution is intimately related to the hypergeometric functions ${ }_{0} F_{1}$ and ${ }_{1} F_{1}$. Recall that

$$
{ }_{0} F_{1}(c, z)=\sum_{m=0}^{\infty} \frac{z^{m}}{m !(c)_{m}}, \quad z \in \mathbb{C}, \quad-c \notin \mathbb{N},
$$

and

$$
{ }_{1} F_{1}(a, c ; z)=\sum_{k=0}^{\infty} \frac{(a)_{k}}{(c)_{k}} \frac{z^{k}}{k !}, \quad z \in \mathbb{C}, \quad-c \notin \mathbb{N} .
$$

The latter is known as the confluent hypergeometric function of the first kind. It is known that (Lebedev, 1972, p.267)

$$
e^{-z}{ }_{1} F_{1}(a, c ; z)={ }_{1} F_{1}(c-a, c ;-z) .
$$

Suppose that

$$
S=\sum_{k=1}^{N} X_{k}, \quad X_{k} \sim \exp (1), \quad N \sim \operatorname{Poisson}(\lambda) .
$$

First, the characteristic function of $S$ is

$$
\mathbb{E}\left(e^{i u S}\right)=\exp \left[\lambda\left(\frac{1}{1-i u}-1\right)\right]={ }_{1} F_{1}(1,1, \lambda i u /(1-i u)) .
$$

Next, the compound Poisson/exponential distribution is of mixed type, but we may calculate derivative of the distribtution function explicitly for $x>0$ :

$$
\begin{aligned}
\frac{\partial}{\partial x} \mathbb{P}\{S \leq x\} & =\sum_{n=1}^{\infty} \frac{e^{-\lambda} \lambda^{n}}{n !} \frac{x^{n-1} e^{-x}}{(n-1) !} \\
& =\lambda e^{-\lambda-x} \sum_{m=0}^{\infty} \frac{(\lambda x)^{m}}{m !(m+1) !}=\lambda e^{-\lambda-x}{ }_{0} F_{1}(2, \lambda x) .
\end{aligned}
$$


Other authors have expressed this in terms of Bessel functions (probably because the name "Bessel" sounds better than "F01"), but one might argue that hypergeometric functions are more natural here. Next, turn to expectations of payoffs $g_{3}$ and $g_{4}$ : if $K>0$,

$$
\begin{aligned}
& \mathbb{E}(X-K)_{+}=\lambda e^{-\lambda} \int_{K}^{\infty} e^{-x}{ }_{0} F_{1}(2, \lambda x) d x \\
& \mathbb{E}(K-X)_{+}=K e^{-\lambda}+\lambda e^{-\lambda} \int_{0}^{K} e^{-x}{ }_{0} F_{1}(2, \lambda x) d x .
\end{aligned}
$$

Finally, the Mellin transform of $S$ is, for $r>0$,

$$
\begin{aligned}
\mathbb{E}\left(S^{r}\right) & =\sum_{n=1}^{\infty} \frac{\lambda^{n} e^{-\lambda}}{n !} \mathbb{E}\left(X_{1}+\cdots+X_{n}\right)^{r} \\
& =\sum_{n=1}^{\infty} \frac{\lambda^{n} e^{-\lambda}}{n !} \int_{0}^{\infty} \frac{x^{r+n-1} e^{-x}}{\Gamma(n)} d x \\
& =\lambda e^{-\lambda} \sum_{m=0}^{\infty} \frac{\lambda^{m}}{(m+1) !} \frac{\Gamma(m+1+r)}{m !} \\
& =\lambda e^{-\lambda} \Gamma(1+r) \sum_{m=0}^{\infty} \frac{\Gamma(m+1+r)}{\Gamma(1+r)} \frac{\Gamma(2)}{\Gamma(m+2)} \frac{\lambda^{m}}{m !} \\
& =\lambda e^{-\lambda} \Gamma(1+r){ }_{1} F_{1}(1+r, 2 ; \lambda) .
\end{aligned}
$$

We can thus write

$$
\mathbb{E}\left(S^{r}\right)=\lambda \Gamma(1+r){ }_{1} F_{1}(1-r, 2 ;-\lambda) .
$$

Observe that the integral moments $\mathbb{E}\left(S^{k}\right), k=1,2, \ldots$, form an infinite series in (4.1), but that they are a finite one in (4.2): if $k=0,1, \ldots$,

$$
\mathbb{E}\left(S^{k}\right)=\lambda \Gamma(1+k)_{1} F_{1}(1-k, 2 ;-\lambda)=\lambda k ! \sum_{j=0}^{k-1} \frac{(1-k)_{j}}{(j+1) !} \frac{(-\lambda)^{j}}{j !} .
$$

We computed $\mathbb{E}(1-S)_{+}, \lambda=1$, by conditioning on $N$ and also with the Mellin inversion formula. The latter was quicker, the results identical. 


\subsection{Generalized Pareto}

In this case there are closed form expressions, in terms of special functions, for the expected payoffs $\mathbb{E}(K-S)_{+}$and $\mathbb{E}(S-K)_{+}$, as well as for the Fourier and Mellin transforms of $S$.

For $a>0$, let

$$
B(a, b ; y)=\int_{0}^{y} x^{a-1}(1-x)^{b-1} d x .
$$

This is the incomplete beta function. For $a, b>0, B(a, b)=B(a, b ; 1)$ is the beta function.

If $\alpha, \beta>0$, we write $Y \sim$ Generalized $\operatorname{Pareto}(\alpha, \beta)$ if the density function of $Y$ is

$$
f_{Y}(x)=\frac{1}{B(\alpha, \beta)} \frac{x^{\beta-1}}{(1+x)^{\alpha+\beta}} I_{\{x>0\}} .
$$

(The usual $\operatorname{Pareto}(\alpha)$ is thus Generalized $\operatorname{Pareto}(\alpha, 1)$.) Then

$$
\begin{aligned}
B(\alpha, \beta) \mathbb{E}(K & -Y)_{+} \\
& =\int_{0}^{K}(K-x) \frac{x^{\beta-1}}{(1+x)^{\alpha+\beta}} d x \\
& =K \int_{1 /(K+1)}^{1} y^{\alpha-1}(1-y)^{\beta-1} d y-\int_{1 /(K+1)}^{1} y^{\alpha-2}(1-y)^{\beta} d y \\
& =K \int_{0}^{K /(K+1)} u^{\beta-1}(1-u)^{\alpha-1} d u-\int_{0}^{K /(K+1)} u^{\beta}(1-u)^{\alpha-2} d u \\
& =K B\left(\beta, \alpha ; \frac{K}{K+1}\right)-B\left(\beta+1, \alpha-1 ; \frac{K}{K+1}\right) .
\end{aligned}
$$

Similarly,

$$
\begin{aligned}
B(\alpha, \beta) \mathbb{E}(Y-K)_{+} & \\
& =(K+1) B\left(\alpha-1, \beta+1, \frac{1}{K+1}\right)-K B\left(\alpha-1, \beta, \frac{1}{K+1}\right) .
\end{aligned}
$$

The Mellin transform of the Generalized Pareto $(\alpha, \beta)$ distribution is

$$
\mathbb{E}\left(Y^{r}\right)=\frac{B(\alpha-r, \beta+r)}{B(\alpha, \beta)}=\frac{\Gamma(\alpha-r) \Gamma(\beta+r)}{\Gamma(\alpha) \Gamma(\beta)},
$$


while its Fourier transform is

$$
\mathbb{E}\left(e^{i u Y}\right)=\frac{1}{B(\alpha, \beta)} \int_{0}^{\infty} \frac{e^{i u x} x^{\beta-1}}{(1+x)^{\alpha+\beta}} d x=\frac{\Gamma(\alpha+\beta)}{\Gamma(\alpha)} \Psi(\beta, 1-\alpha ;-i u) .
$$

This characteristic function may be expressed in terms of the expontial integral function or the incomplete gamma functions (see Section 3.2.2).

As an illustration, we compute the excess-of-loss (XL) premium $\mathbb{E}(X-$ $K)_{+}$if $X \sim \operatorname{Pareto}(\alpha)$, using a polynomial damping factor. Let $\beta=3$ and $\alpha=5$. Then the XL payoff function and Pareto claim severity density have the following Fourier transform, for $u \in \mathbb{R}$ :

$$
\begin{aligned}
\widehat{g^{[-3]}}(u) & =\left[1-(K+1) \frac{i u}{2}\right]\left[\frac{e^{i u K}}{(1+K)}+i u e^{-i u} E_{1}(-i u(K+1))\right]-\frac{e^{i u K}}{2(1+K)} \\
\widehat{\nu_{X}^{[3]}}(u) & =\frac{5}{2}\left[1+i u-u^{2} e^{-i u} E_{1}(-i u)\right] .
\end{aligned}
$$

Now the XL premium $\mathbb{E}(X-K)_{+}=\int_{-\infty}^{\infty} \widehat{g^{[-3]}}(u) \widehat{\nu_{X}^{[3]}}(u) d u$ is obtained by evaluating the integral numerically. This can easily be done in Excel (see next section for further details).

\subsection{Compound Poisson/Pareto}

In this example and the next one there is no explicit formula for stop-loss premiums. Our Fourier inversion formulas will be compared to simulation results.

Consider a stop-loss (SL) premium on aggregate claims. Here different i.i.d. random variables $X_{j}$, with a common distribution, represent individual claim severities. These are summed to form a compound Poisson distribution:

$$
S=\sum_{j=1}^{N} X_{j},
$$

where $N \sim \operatorname{Poisson}(\lambda) . N$ is assumed independent of the individual claim severities $\left\{X_{j}\right\}$. The compound Poisson variable $S$ represents the aggregate claims over some time interval.

Suppose we want to compute an SL premium for the aggregate claims variable $S$ :

$$
\mathbb{E}(S-K)_{+}=\int_{0}^{\infty} g(x) d \mu_{S}(x)
$$


where $\mu_{S}$ is the distribution of $S$ and $g(x)=(x-K)_{+}$. As before, this can be written as

$$
\mathbb{E}(S-K)_{+}=\int_{0}^{\infty} g^{[-\beta]}(x) d \mu_{X}^{[\beta]}(x),
$$

where $g^{[-\beta]}(x)=(1+x)^{-\beta} g(x)$ and $\mu_{X}^{[\beta]}(d x)=(1+x)^{\beta} \mu_{X}(d x)$.

By Theorem 3.3, the SL premium is also equal to

$$
\mathbb{E}(S-K)_{+}=\frac{1}{2 \pi} \int_{-\infty}^{\infty} \widehat{g^{[-\beta]}}(-u) \widehat{\mu_{S}^{[\beta]}}(u) d u .
$$

We need to calculate

$$
\widehat{\mu_{S}^{[\beta]}}(u)=\sum_{j=0}^{\beta}\left(\begin{array}{l}
\beta \\
j
\end{array}\right)(-i)^{j} \frac{d^{j}}{d u^{j}} e^{\lambda\left[\hat{\mu}_{X}(u)-1\right]}, \quad u \in \mathbb{R} .
$$

The derivatives $\hat{\nu}_{X}^{(j)}$ take the form

$$
\hat{\nu}_{X}^{(j)}(u)=\int_{0}^{\infty} e^{i u x} \frac{\alpha(i x)^{j}}{(1+x)^{\alpha+1}} d x, \quad u \in \mathbb{R}, \quad j=0,1, \ldots, \alpha .
$$

Using integration by parts, they can be written in terms of the exponential integral function as in Section 3.2.2. The same section gives $\widehat{g^{[-3]}}$, while, for $u \in \mathbb{R}$,

$$
\begin{aligned}
\widehat{\mu_{S}^{[3]}}(u)= & \hat{\mu}_{S}(u)-3 i \hat{\mu}_{S}^{\prime}(u)-3 \hat{\mu}_{S}^{\prime \prime}(u)+i \hat{\mu}_{S}^{\prime \prime \prime}(u) \\
= & e^{\lambda\left[\hat{\mu}_{X}(u)-1\right]}\left\{1-3 i \lambda \hat{\mu}_{X}^{\prime}(u)-3\left[\lambda \hat{\mu}_{X}^{\prime \prime}(u)+\lambda^{2}\left(\hat{\mu}_{X}^{\prime}(u)\right)^{2}\right]\right. \\
& \left.+i\left[\lambda \hat{\mu}_{X}^{\prime \prime \prime}(u)+3 \lambda^{2} \hat{\mu}_{X}^{\prime}(u) \hat{\mu}_{X}^{\prime \prime}(u)+\lambda^{3}\left(\hat{\mu}_{X}^{\prime}(u)\right)^{3}\right]\right\} .
\end{aligned}
$$

Table 1 lists the results for different values of the Poisson parameter $\lambda$ and the retention limit $K$. These are compared with simulated SL premiums based on 100,000,000 replicated samples.

Table 2 lists additional SL premiums, also computed from (4.4), by letting vary the Poisson parameter $\lambda$ and the Pareto parameter $\alpha$ such that the expected value of $S$ is always 1 . Again, these are compared with the simulated SL premiums obtained from 100,000,000 replicated samples.

These numbers were obtained with Romberg's numerical integration method, coded in Visual Basic and implemented in Excel. The program is can be downloaded at http://www. mathstat. concordia.ca . 
Table 1: SL premiums - compound Poisson/Pareto

\begin{tabular}{|c|cc|cc|cc|}
\hline$\lambda$ & \multicolumn{2}{|c|}{1} & \multicolumn{2}{c|}{2} & \multicolumn{2}{c|}{3} \\
\hline$K$ & Simulated & Integral & Simulated & Integral & Simulated & Integral \\
\hline 0.25 & 0.1364 & 0.1342 & 0.3224 & 0.3215 & 1.0096 & 1.0095 \\
0.5 & 0.0281 & 0.0283 & 0.2068 & 0.2063 & 0.7938 & 0.7937 \\
1 & 0.0776 & 0.0764 & 0.0863 & 0.0862 & 0.4617 & 0.4614 \\
\hline
\end{tabular}

Table 2: SL premiums - compound Poisson/Pareto

\begin{tabular}{|c|cc|cc|cc|}
\hline$\lambda$ & \multicolumn{2}{|c|}{1} & \multicolumn{2}{c|}{2} & \multicolumn{2}{c|}{3} \\
\hline$K$ & Simulated & Integral & Simulated & Integral & Simulated & Integral \\
\hline 1 & 0.305 & 0.3049 & 0.2703 & 0.2701 & 0.2448 & 0.2446 \\
2 & 0.079 & 0.07894 & 0.0526 & 0.05226 & 0.036 & 0.0358 \\
3 & 0.0213 & 0.02126 & 0.0098 & 0.00971 & 0.0046 & 0.00453 \\
\hline
\end{tabular}

\subsection{Compound Poisson/Pareto plus $\alpha$-stable}

Now let us consider the SL premium $\mathbb{E}(Z-K)_{+}$for a more general aggregate claims distribution,

$$
Z=S+J,
$$

where $S$ is compound Poisson

$$
S=\sum_{j=1}^{N} Y_{j},
$$

the claims $Y_{j}$ having density

$$
f_{Y}(y)=\frac{\alpha}{y^{1+\alpha}} I_{\{y>1\}} .
$$

(With our previous notation, this means $Y_{j}-1 \sim \operatorname{Pareto}(\alpha)$ ). Let $J$ have an $\alpha$-stable distribution with Lévy measure

$$
\nu_{Z}(d y)=\frac{\lambda}{y^{1+\alpha}} I_{(0, \infty)}(y) d y .
$$


Furrer (1998) considered a risk model under which aggregate claims had the same distribution as $S+J$.

It is known that

$$
\hat{\mu}_{Z}(u)=e^{-\Psi_{Z}(u)},
$$

where $\Psi_{Z}$ is the Lévy-Khintchine exponent of $Z$, given by

$$
\Psi_{Z}(u)=\int_{0}^{\infty}\left(1-e^{i u x}+i u x\right) \mu_{Z}(d x)=\sigma^{\alpha}|u|^{\alpha}[1-i \operatorname{sign}(u) \tan (\alpha \pi / 2)]
$$

$\sigma$ being the variance of $Z$ ( $\sigma$ is a function of $\lambda$, see Furrer (1998)).

\section{References}

[1] Abramowitz, M., and Stegun, I. (1970). Handbook of Mathematical Functions: With Formulas, Graphs and Mathematical Tables. Dover.

[2] Apostol, T.M. (1974). Mathematical Analysis, Second Edition. AddisonWesley, Reading, Mass.

[3] Bakshi, G., and Madan, D.B. (2000). Spanning and Derivative-security Valuation. J. of Financial Economics 55: 205-238.

[4] Carr, P., and Madan, D.B. (1999). Option valuation using the fast Fourier transform. J. Computational Finance 2: 61-73.

[5] Furrer, H.J. (1998). Risk processes perturbed by a $\alpha$-stable Lévy motion. Scand. Actuarial Journal 1998: 59-74.

[6] Heston, S.L. (1993). A closed-form solution for options with stochastic volatility with application to bond and currency options. Rev. Fin. Studies 6: $327-343$.

[7] Kendall, M., and Stuart, A. (1977). The Advanced Theory of Statistics, Fourth Edition. Griffin, London.

[8] Lebedev, N.N. (1972). Special Functions and their Applications. Dover, New York.

[9] Lee, R.W. (2004). Option pricing by transform methods: extensions, unification, and error control. Journal of Computational Finance 7:51-86. 
[10] Lewis, A.L. (2001). A simple option formula for general jump-diffusion and other exponential Lévy processes. Unpublished. OptionCity. net publications: http://optioncity.net/pubs/ExpLevy ·pdf.

[11] Lukacs, E. (1970). Characteristic Functions, Fourth Edition. Griffin, London.

[12] Malliavin, P. (1995). Integration and Probability. Springer Verlag, New York.

[13] Raible, S. (2000). Lévy Processes in Finance: Theory, Numerics, and Empirical Facts. PhD Dissertation, Faculty of Mathematics, University of Freiburg, Germany.

[14] Rudin, W. (1987). Real and Complex Analysis, Third Edition. McGrawHill, New York.

[15] Titchmarsh, E.C. (1975). Introduction to the Theory of Fourier Integrals. Oxford University Press.

\section{A The Fourier transform}

In this appendix we state some essential definitions and results related to Fourier transforms. For $1 \leq p<\infty$ let $L^{p}$ denote the space of measurable functions $h: \mathbb{R} \mapsto \mathbb{C}$ such that $\int_{\mathbb{R}}|h(x)|^{p} d x<\infty$; the space $L^{\infty}$ consists in the functions from $\mathbb{R}$ to $\mathbb{C}$ which are essentially bounded. Let $\mu$ be a signed measure on $(\mathcal{B}(\mathbb{R}), \mathbb{R})$ with $|\mu|<\infty$, and define its Fourier transform as

$$
\hat{\mu}(u)=\int_{-\infty}^{\infty} e^{i u x} \mu(d x) .
$$

Since $\left|e^{i u x}\right|=1$, this is an ordinary (that is, proper) Lebesgue integral. If $h \in L^{1}$, then the Fourier transform of $h$ is defined as

$$
\hat{h}(u)=\int_{-\infty}^{\infty} e^{i u x} h(x) d x, \quad u \in \mathbb{R} .
$$

Once again, because $h$ is assumed integrable, the above is an ordinary integral. The convolution of a real function $h$ with a signed measure $\mu$ is defined as

$$
\left(\tau_{\mu} h\right)(x)=\int_{-\infty}^{\infty} h(x-y) \mu(d y)
$$


(when the integral exists), and the convolution of two real functions $h, k$ as

$$
(h * k)(x)=\int_{-\infty}^{\infty} h(x-y) k(y) d y
$$

(when the integral exists). It is known (Malliavin, 1995, p.114) that

Theorem A.1 If $\mu$ is a signed measure with $|\mu|<\infty$, and $h \in L^{p}$ (for some $1 \leq p \leq \infty)$, then $\int h(x-y) \mu(d y)$ converges almost everywhere and $\tau_{\mu} h \in L^{p}$.

A consequence is that if $h, k \in L^{1}$, then $\int h(x-y) k(y) d y$ converges almost everywhere and $h * k \in L^{1}$. For the next result, see Malliavin (1995, p.107).

Theorem A.2 If $\mu$ is a signed measure with $|\mu|<\infty$ and $h \in L^{1}$, then $\widehat{\tau_{\mu} h}=\hat{h} \hat{\mu}$.

A consequence is that if $h, k \in L^{1}$, then $\widehat{h * k}=\hat{h} \hat{k}$.

\section{Theorem A.3 (Malliavin, 1995, pp.130, 134)}

(a) If $h, \hat{h} \in L^{1}$, then

$$
h(x)=\frac{1}{2 \pi} \int_{-\infty}^{\infty} e^{-i u x} \hat{h}(u) d u \quad \text { for almost all } x \in \mathbb{R},
$$

and $h, \hat{h} \in L^{p}$ for all $1 \leq p \leq \infty$. The right-hand side is a continuous function of $x$, and so the above identity holds for all $x$ if $h$ is continuous.

(b) If $h, \hat{h} \in L^{1}$ and $\mu$ is a signed measure with $|\mu|<\infty$, then Parseval's identity holds:

$$
\int_{-\infty}^{\infty} h(x) \mu(d x)=\frac{1}{2 \pi} \int_{-\infty}^{\infty} \hat{h}(u) \hat{\mu}(-u) d u .
$$

This is a fairly restrictive result. For instance, the Fourier transform of the normal density function with mean $m$ and variance $s^{2}$ is

$$
\hat{h}_{1}(u)=e^{i u m-s^{2} u^{2} / 2},
$$


which is in $L^{1}$; as Theorem A.3 says, both $h_{1}$ and $\hat{h}_{1}$ are uniformly bounded almost everywhere. The same holds for the double exponential density. However, the Fourier transform of the exponential density function with mean $1 / \lambda$ is

$$
\hat{h}_{2}(u)=\frac{\lambda}{\lambda-i u},
$$

which is not in $L^{1}$, and Theorem A.3 does not apply. A less restrictive inversion theorem is thus required for the applications considered in this paper.

One extension of Theorem A.3 is Plancherel's Theorem (see Malliavin, 1995, p.132, and Rudin, 1987, p.186), which applies to square-integrable functions. (We do not need Plancherel's Theorem in this paper, but other authors have applied it to derivative pricing.)

Theorem A.4 The Fourier transform has an extension to functions in $L^{2}$ which satisfies:

(a) For every $h \in L^{2},\|h\|^{2}=(2 \pi)^{-1}\|\hat{h}\|^{2}$.

(b) If

$$
\begin{aligned}
& \qquad \phi_{M}(u)=\int_{-M}^{M} h(x) e^{i x u} d x, \quad \psi_{M}(u)=\frac{1}{2 \pi} \int_{-M}^{M} \hat{h}(u) e^{-i x u} d u, \\
& \text { then }\left\|\phi_{M}-\hat{h}\right\| \rightarrow 0 \text { and }\left\|\psi_{M}-h\right\| \rightarrow 0 \text { as } M \rightarrow \infty .
\end{aligned}
$$

For a function $h$ which is in $L^{2}$ but not in $L^{1}$, we see that the (extension of) the Fourier transform is the limit of $\phi_{M}(u)$ as $M$ tends to infinity. This type of integral, which may exist even though the integrand is not absolutely integrable over $\mathbb{R}$, is called a "principal value" integral (or "Cauchy principal value"). We will use the notation

$$
P V \int_{-\infty}^{\infty} k(x) d x=\lim _{M \rightarrow \infty} \int_{-M}^{M} k(x) d x
$$

when the limit exists.

The inversion theorem needed for our results is the following. It only requires that $h \in L^{1}$.

Theorem A.5 (Apostol, 1974, p.324) Suppose $h$ is a real function which satisfies the following conditions: 
(a) $h \in L^{1}$ and

(b) either (b1) or (b2) holds:

(b1) $h(x+)$ and $h(x-)$ both exist and the integrals below are finite for some $\epsilon>0$ :

$$
\int_{0}^{\epsilon} \frac{h(x+t)-h(x+)}{t} d t, \quad \int_{-\epsilon}^{0} \frac{h(x-t)-h(x-)}{t} d t
$$

(b2) $h(x)$ has bounded variation in some open neighborhood of $x$. (This implies that $h(x+)$ and $h(x-)$ both exist. A sufficient condition for a function to have bounded variation is having a derivative.)

Then

$$
\frac{1}{2}[h(x+)+h(x-)]=\frac{1}{2 \pi} P V \int_{-\infty}^{\infty} e^{-i u x} \hat{h}(u) d u .
$$

(In case where $\hat{h} \in L^{1}$ we know that the last integral converges absolutely, and is therefore an ordinary Lebesgue integral.)

We are now able to extend Parseval's identity (Theorem A.3(b)). (Another reference for Parseval's theorem is see for instance Titchmarsh, 1975, Theorem 39.) Let $\mu$ be a signed measure with $|\mu|<\infty$ and $h \in L^{1}$, and suppose that the convolution

$$
y \mapsto\left(\tau_{\mu} h\right)(y)=\int_{-\infty}^{\infty} h(y-x) \mu(d x)
$$

satisfies assumption (b) of Theorem A.5, and that moreover $\left(\tau_{\mu} h\right)(y)$ is continuous at $y=0$. It can be seen that $\tau_{\mu} h \in L^{1}$ (from Theorem A.1). Theorem A.5 then yields

$$
\left(\tau_{\mu} h\right)(0)=\int_{-\infty}^{\infty} h(-x) \mu(d x)=\frac{1}{2 \pi} P V \int_{-\infty}^{\infty} \hat{h}(u) \hat{\mu}(u) d u .
$$

To get Parseval's identity, rewrite this by replacing $h(x)$ with $g(-x)$, after noting that

$$
\int_{-\infty}^{\infty} e^{i u x} g(-x) d x=\hat{g}(-u)
$$

to get

$$
\int_{-\infty}^{\infty} g(x) \mu(d x)=\frac{1}{2 \pi} P V \int_{-\infty}^{\infty} \hat{g}(-u) \hat{\mu}(u) d u .
$$

We state the result obtained as a theorem. 
Theorem A.6 Let $\mu_{X}$ be the distribution of a variable $X$ (that is, $\mu_{X}$ is the measure on $\mathbb{R}$ induced by the distribution function $\left.F_{X}(x)=\mathbb{P}\{X \leq x\}\right)$. Suppose that (i) $g \in L^{1}$, (ii) the function $y \mapsto \mathbb{E} g(y+X)$ is continuous at $y=0$ and (iii) $g$ satisfies condition (b) of Theorem A.5.

Then

$$
\mathbb{E} g(X)=\int_{-\infty}^{\infty} g(x) \mu_{X}(d x)=\frac{1}{2 \pi} P V \int_{-\infty}^{\infty} \hat{g}(-u) \hat{\mu}_{X}(u) d u .
$$

\section{B Proofs of theorems}

Proof of Theorem 2.2. Let $g(x)=I_{(a, a+h]}(x)$. Then

$$
\mathbb{E} g(X)=F_{X}(a+h)-F_{X}(a)
$$

and the conditions of Lemmas 2.1 and 2.2(a) are satisfied. We may thus apply Theorem 2.1 with $\alpha=0$. The result follows from

$$
\hat{g}(u)=\frac{e^{i u(a+h)}-e^{i u a}}{i u} .
$$

Proof of Theorem 2.3. This formula is equivalent to

$$
1-F_{X}(b)=\frac{1}{2}+\frac{1}{2 \pi} \int_{0}^{\infty} \frac{1}{i u}\left[e^{-i u b} \hat{\nu}_{X}(u)-e^{i u b} \hat{\nu}_{X}(-u)\right] d u .
$$

We prove the latter for $b=0$; a translation $X \rightarrow X-b$ then finishes the proof.

If we let $g(x)=I_{(0, \infty)}(x)$, then $\hat{g}(u)=\int_{0}^{\infty} e^{i u x} d x$ does not exist for real $u$, but, if $\operatorname{Im}(z)>0$,

$$
\hat{g}(z)=\int_{0}^{\infty} e^{i z x} d x=-\frac{1}{i z} .
$$

We thus apply Theorem 2.1: temporarily assume there exists $\alpha>0$ such that $\mathbb{E}\left(e^{\alpha X}\right)<\infty$; then

$$
1-F_{X}(0)=\mathbb{E} g(X)=\frac{1}{2 \pi} P V \int_{-\infty}^{\infty} \hat{g}(-u+i \alpha) \hat{\nu}_{X}(u-i \alpha) d u
$$

Let

$$
h(z)=\hat{g}(-z) \hat{\nu}_{X}(z)=\frac{\mathbb{E}\left(e^{i z X}\right)}{i z} .
$$


The function $h$ is analytic in $\{z \mid-\alpha<\operatorname{Im}(z)<0\}$ and has a pole at $z=0$. Hence,

$$
\int_{C_{M, \epsilon}} h(z) d z=0
$$

where $C_{M, \epsilon}$ is the closed path in Figure 1.

We know that $\mathbb{E} g(X)$ equals the integral of $h(z)$ on the line

$$
\{z \mid \operatorname{Im}(z)=-\alpha\}
$$

We also know that, for $0 \leq y \leq \alpha$,

$$
\left|\mathbb{E} e^{i(M-i y) X}\right| \leq \mathbb{E}\left(e^{y X}\right) \leq \mathbb{P}\{X \leq 0\}+\mathbb{E} e^{\alpha X} I_{\{X>0\}}=C<\infty .
$$

Hence, on the segment $\{z \mid \operatorname{Re}(z)=M,-\alpha \leq \operatorname{Im}(z) \leq 0\}$,

$$
|h(z)| \leq\left|\frac{C}{i z}\right| \leq \frac{C}{M}
$$

and so

$$
\left|\int_{M-i \alpha}^{M} h(z) d z\right| \leq \frac{\alpha C}{M} \rightarrow 0
$$

as $M \rightarrow \infty$. In the same way,

$$
\left|\int_{-M-i \alpha}^{-M} h(z) d z\right| \rightarrow 0
$$

as $M \rightarrow \infty$. We conclude that

$$
P V \int_{-\infty}^{\infty} \hat{g}(-u+i \alpha) \hat{\nu}_{X}(u-i \alpha) d u=\lim _{M \rightarrow \infty} \int_{L_{M, \epsilon}} h(z) d z,
$$

where $L_{M, \epsilon}$ is the path going along the real axis from $-\infty$ to $-\epsilon$, then around the half-circle $R_{\epsilon}$ (Figure 1), then on the real axis from $\epsilon$ to $+\infty$.

Next, let $\epsilon \rightarrow 0+$. The integral over the half-circle in the path $L_{M, \epsilon}$ is (since $d z=\epsilon i e^{i \theta} d \theta$ )

$$
\int_{-\pi}^{0} h\left(\epsilon e^{i \theta}\right) \epsilon i e^{i \theta} d \theta=\int_{-\pi}^{0} \hat{\nu}_{X}\left(\epsilon e^{i \theta}\right) d \theta \rightarrow \pi \quad \text { as } \epsilon \rightarrow 0+.
$$


This implies

$$
\begin{aligned}
1-F_{X}(0) & =\frac{1}{2}+\lim _{M \rightarrow \infty} \frac{1}{2 \pi} \int_{0}^{M}[h(u)-h(-u)] d u \\
& =\frac{1}{2}+\lim _{M \rightarrow \infty} \frac{1}{2 \pi} \int_{0}^{M} \frac{1}{i u}\left[\mathbb{E}\left(e^{i u X}\right)-\mathbb{E}\left(e^{-i u X}\right)\right] d u \\
& =\frac{1}{2}+\lim _{M \rightarrow \infty} \frac{1}{\pi} \int_{0}^{M} \frac{1}{u} \mathbb{E}[\sin (u X)] d u
\end{aligned}
$$

If we then let $X^{\prime}=X-b$, we find

$$
\mathbb{E}\left(e^{i u X^{\prime}}\right)=e^{-i u b} \mathbb{E}\left(e^{i u X}\right)
$$

and so

$$
\begin{aligned}
1-F_{X}(b) & =1-F_{X^{\prime}}(0) \\
& =\frac{1}{2}+\lim _{M \rightarrow \infty} \frac{1}{2 \pi} \int_{0}^{M}\left[e^{-i u b} \hat{\nu}_{X}(u)-e^{i u b} \hat{\nu}_{X}(-u)\right] d u .
\end{aligned}
$$

If $\mathbb{E}\left(e^{\alpha X}\right)<\infty$ for some $\alpha>0$, then we are finished. If not, then consider $X^{a}=X \wedge a$, where $a>0$. Formula (B.1) holds for $X^{a}$, and so

$$
1-F_{X}(0)=1-F_{X^{a}}(0)=\frac{1}{2}+\lim _{M \rightarrow \infty} \frac{1}{\pi} \int_{0}^{M} \frac{1}{u} \mathbb{E}\left[\sin \left(u X^{a}\right)\right] d u .
$$

The result is proved if it is shown that

$$
\lim _{M \rightarrow \infty}\left[\int_{0}^{M} \frac{1}{u} \mathbb{E}[\sin (u X)] d u-\int_{0}^{M} \frac{1}{u} \mathbb{E}\left[\sin \left(u X^{a}\right)\right] d u\right]=0 .
$$

The expression in square brackets is equal to

$\mathbb{E}\left\{\left[\int_{0}^{M X} \frac{\sin (y)}{y} d y-\int_{0}^{M a} \frac{\sin (y)}{y} d y\right] I_{\{X>a\}}\right\}=\mathbb{E}\left[\int_{M a}^{M X} \frac{\sin (y)}{y} d y I_{\{X>a\}}\right]$,

which tends to 0 as $M \rightarrow \infty$, by dominated convergence. 
Proof of Theorem 3.1. (a) Let $K=e^{c}$. First, assume that $\mathbb{P}\{S=0\}=0$. If $g(x)=\left(e^{c}-e^{x}\right)_{+}$and $z \in \mathbb{C}$,

$$
\begin{aligned}
\hat{g}(z) & :=\int_{-\infty}^{c} e^{i z x}\left(e^{c}-e^{x}\right) d x \\
& =\lim _{M \rightarrow \infty} \int_{-M}^{c}\left(e^{i z x+c}-e^{(i z+1) x}\right) d x \\
& =\lim _{M \rightarrow \infty}\left\{e^{c}\left[\left.\frac{e^{i z x}}{i z}\right|_{-M} ^{c}\right]-\left[\left.\frac{e^{(i z+1) x}}{(i z+1)}\right|_{-M} ^{c}\right]\right\} \\
& =e^{(i z+1) c}\left(\frac{1}{i z}-\frac{1}{i z+1}\right)+\lim _{M \rightarrow \infty}\left(\frac{1}{i z+1} e^{-(i z+1) M}-\frac{1}{i z} e^{-i z M+c}\right) .
\end{aligned}
$$

The limit exists, and equals 0 , if and only if, $\operatorname{Im}(z)<0$. Hence,

$$
\hat{g}(z)=\frac{e^{(i z+1) c}}{i z(i z+1)}, \quad \operatorname{Im}(z)<0 .
$$

(This formula is in Lewis (2001).)

Let $h(z)=\hat{g}(-z) \hat{\mu}_{X}(z)$. We need to restrict $z$ to $\operatorname{Im}(z)>0$ for $\hat{g}(-z)$ to exist, and therefore, we need to assume that $\mathbb{E}\left(e^{\alpha X}\right)$ exists for some $\alpha<0$. This proves the first formula in (a), if $\mathbb{P}\{S=0\}=0$.

If $\mathbb{P}\{S=0\}>0$, then define a new variable $S^{*}$ with distribution

$$
\mathbb{P}\left\{S^{*} \in A\right\}=\frac{\mathbb{P}\{S \in A, S>0\}}{\mathbb{P}\{S>0\}} .
$$

Then

$$
\mathbb{E}(K-S)_{+}=K \mathbb{P}\{S=0\}+\mathbb{P}\{S>0\} \mathbb{E}\left(K-S^{*}\right)_{+}
$$

which yields the result, since

$$
\mu_{S^{*}}(u)=\frac{\mu_{S^{*}}(u)}{\mathbb{P}\{S>0\}} .
$$

The second formula in (a) follows from the usual relationship $y_{+}-(-y)_{+}=y$, $y=S-K$.

Part (b) is obtained by first assuming that $\mathbb{P}\{S=0\}=0$ and that there exists $\alpha<0$ such that $\mathbb{E}\left(S^{\alpha}\right)<\infty$. The first formula in part (a) then holds. 
The function $h(z)$ is analytic in the upper complex plane, except for a simple pole at the origin. We have

$$
\mathbb{E}(K-S)_{+}=\int_{-\infty-i \alpha}^{\infty-i \alpha} h(z) d z
$$

where the path of integration is the line $\{z \mid \operatorname{Im}(z)=-\alpha\}$. For $0<\epsilon<M$, define a closed path of integration $C_{M, \epsilon}$ as in Figure 2. The integral of $h(z)$ along $C_{M, \epsilon}$ is 0 .

It is easy to see that

$$
\lim _{M \rightarrow \infty} \int_{-M}^{-M-i \alpha} h(z) d z=\lim _{M \rightarrow \infty} \int_{M}^{M-i \alpha} h(z) d z=0
$$

and so

$$
P V \int_{-\infty}^{\infty} h(u-i \alpha) d u=\int_{R_{\epsilon}} h(z) d z+\left(\int_{-\infty}^{-\epsilon}+\int_{\epsilon}^{\infty}\right) h(z) d z
$$

where $R_{\epsilon}$ is the half-circle around the origin defined above. We find

$$
\begin{aligned}
\lim _{\epsilon \rightarrow 0+} \int_{R_{\epsilon}} h(z) d z & =\lim _{\epsilon \rightarrow 0+} \int_{\pi}^{0} h\left(\epsilon e^{i \theta}\right) \epsilon i e^{i \theta} d \theta \\
& =\lim _{\epsilon \rightarrow 0+} \int_{\pi}^{0} \frac{e^{-i \epsilon e^{i \theta}+c}}{\left(-i \epsilon e^{i \theta}\right)\left(1-i \epsilon e^{i \theta}\right)} i \epsilon e^{i \theta} d \theta \\
& =K \pi .
\end{aligned}
$$

Hence,

$$
\mathbb{E}\left(e^{c}-e^{X}\right)_{+}=\frac{K}{2}+\frac{1}{2 \pi} \int_{0}^{\infty}[h(u)+h(-u)] d u .
$$

Since $h(u)+h(-u)=2 \operatorname{Re}[h(u)]$, we thus have

$$
\mathbb{E}\left(K-e^{X}\right)_{+}=\frac{K}{2}+\frac{1}{\pi} \int_{0}^{\infty} \operatorname{Re}\left[\hat{g}(-u) \hat{\mu}_{X}(u)\right] d u .
$$

This formula was obtained under the assumption that there exists $\alpha<0$ such that $\mathbb{E}\left(e^{\alpha X}\right)<\infty$. If this is not the case, then consider

$$
X^{a}=X \vee(-a),
$$


for $a>0$. As $a \rightarrow \infty, \mathbb{E}\left(e^{i u X^{a}}\right) \rightarrow \mathbb{E}\left(e^{i u X}\right)$ uniformly in $u \in \mathbb{R}$. Since

$$
|\hat{g}(u)| \sim \frac{e^{c}}{u^{2}},
$$

we find that

$$
\mathbb{E}\left(K-e^{X}\right)_{+}=\lim _{a \rightarrow \infty} \mathbb{E}\left(K-e^{X^{a}}\right)_{+}=\frac{K}{2}+\frac{1}{\pi} \int_{0}^{\infty} \operatorname{Re}[\hat{g}(-u) \hat{\mu}(u)] d u
$$

by dominated convergence. Finally, (B.2) holds for all $X$.

The last formula may be proved another way. The function $h(z)$ may be rewritten as

$$
e^{c} \frac{e^{-i z c}}{-i z} \frac{\hat{\nu}_{X}(z)}{1-i z}
$$

Apart from the factor $e^{c}$, this is the Fourier transform of the convolution of an exponential density with the law of $X$. This suggests proceeding as follows:

$$
\mathbb{E}\left(e^{c}-e^{X}\right)_{+}=e^{c} \int_{-\infty}^{c}\left(1-e^{x-c}\right) d \nu_{X}(x)=e^{c} \mathbb{P}\{X+G \leq c\},
$$

if $G \sim \exp (1)$ is independent of $X$. By Theorem 2.3,

$$
\begin{aligned}
e^{c} \mathbb{P}\{X+G \leq c\} & =\frac{e^{c}}{2}+\frac{1}{2 \pi} \int_{0}^{\infty} \frac{e^{c}}{i u}\left[e^{i u c} \frac{\hat{\nu}_{X}(-u)}{1+i u}-e^{-i u c} \frac{\hat{\nu}_{X}(u)}{1-i u}\right] d u \\
& =\frac{K}{2}+\frac{1}{2 \pi} \int_{0}^{\infty}[h(u)+h(-u)] d u
\end{aligned}
$$

which is the same as (B.2). Finally, the formulas in (b) are found by taking into account the cases where $\mathbb{P}\{S=0\}>0$, as in the proof of (a). 
Figure 1

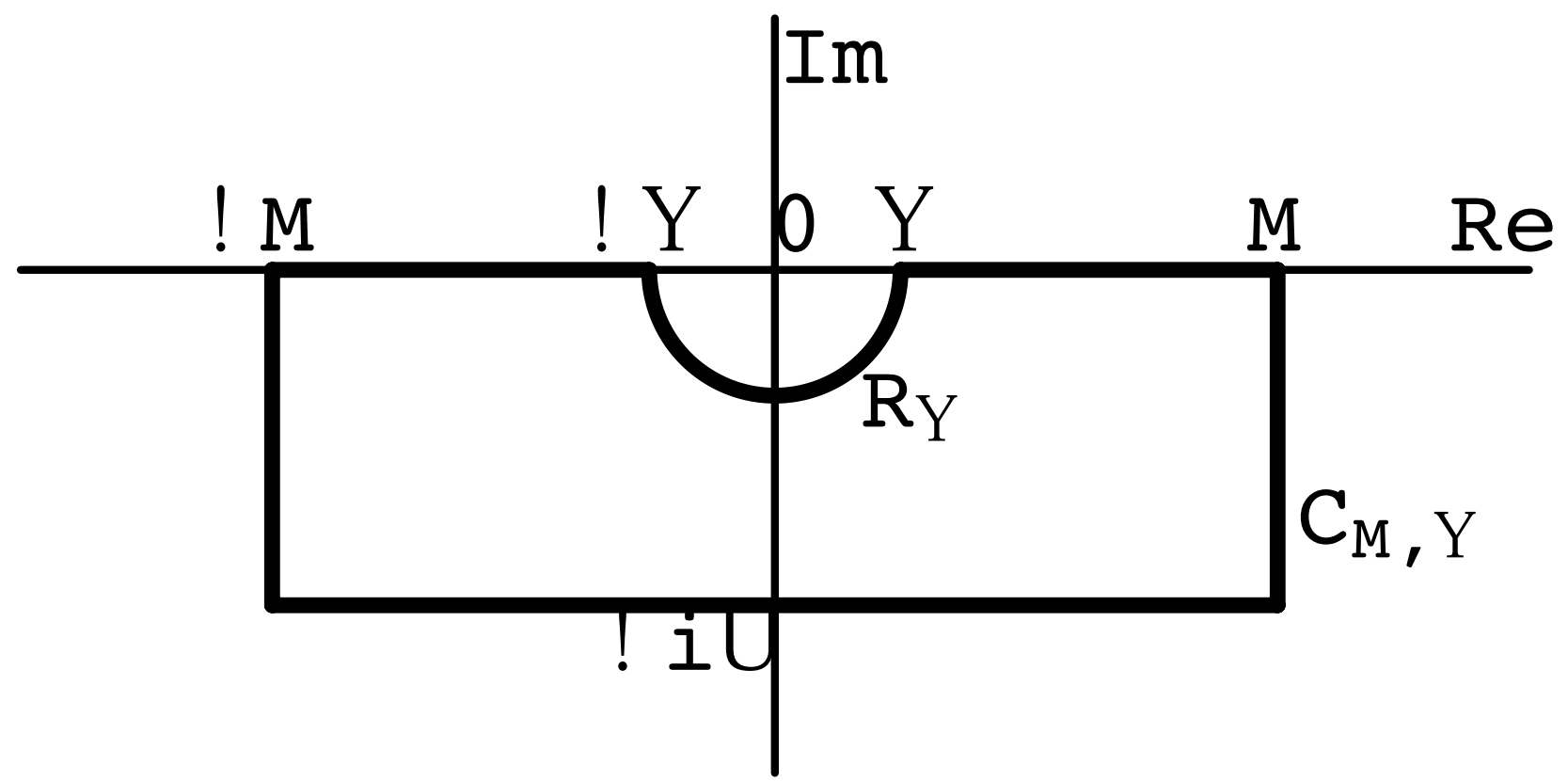

Figure 2

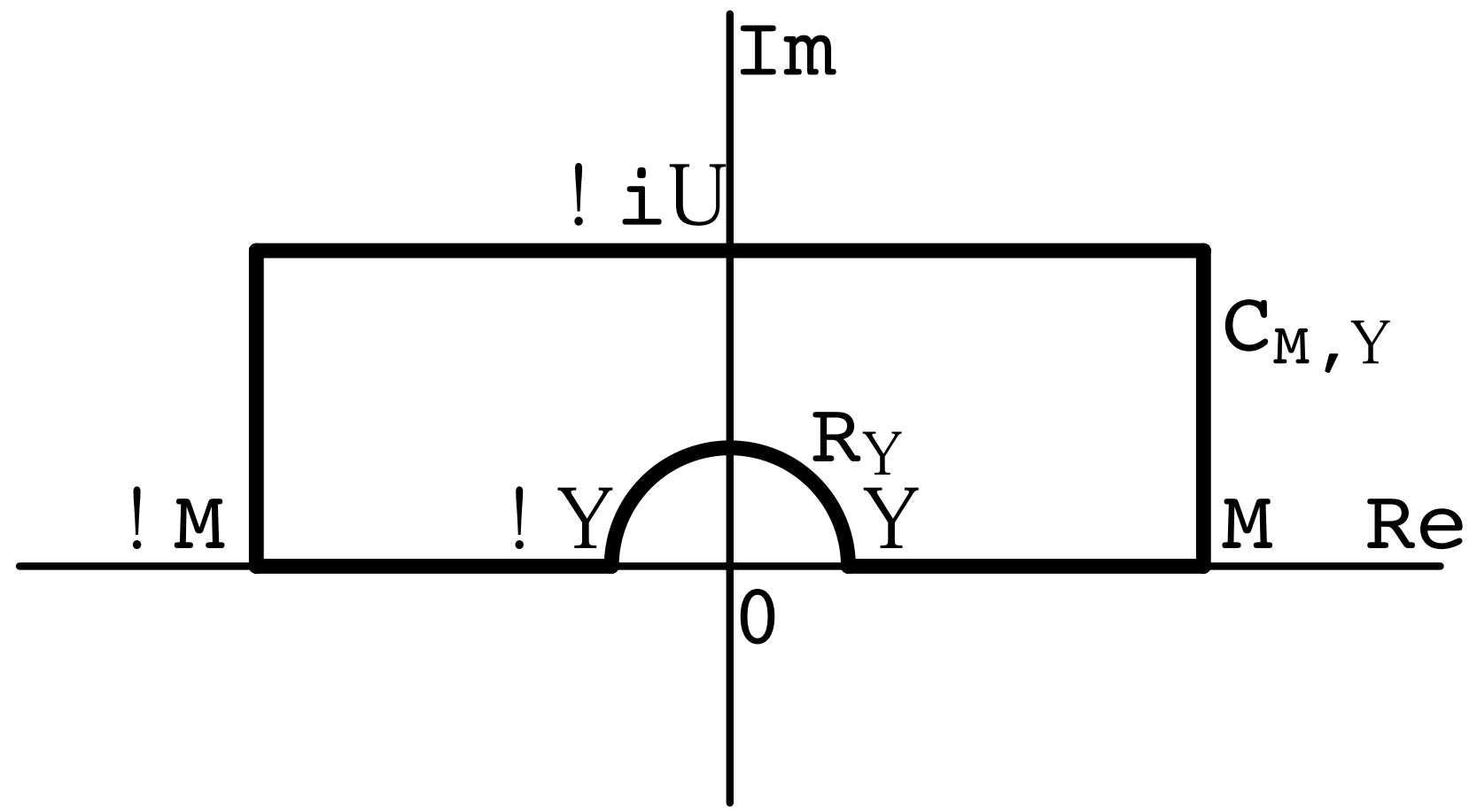




\section{University Library}

\section{- M M I N E R VA A gateway to Melbourne's research publications}

Minerva Access is the Institutional Repository of The University of Melbourne

Author/s:

DUFRESNE, DANIEL;Garrido, Jose;Morales, Manuel

Title:

Fourier inversion formulas in option pricingand insurance

Date:

2005-12

Citation:

Dufresne, Daniel and Garrido, Jose and Morales, Manuel (2005) Fourier inversion formulas in option pricingand insurance.

Persistent Link:

http://hdl.handle.net/11343/34326 